\title{
Clinical Features and Follow-up Results of Sporadic Medullary Thyroid Carcinoma Patients Surgical Treatment
}

\author{
${ }^{1}$ Romanchishen AF, ${ }^{2}$ Lisovsky OV, ${ }^{2}$ Vabalayte KV \\ ${ }^{1}$ Professor of Surgery and Oncology, Department of Hospital Surgery and Oncology of Saint-Petersburg State Pediatric Medical \\ Academy, Saint-Petersburg City Center of Endocrine Surgery and Oncology, Russia \\ ${ }^{2}$ Department of Hospital Surgery and Oncology of Saint-Petersburg State Pediatric Medical Academy, Saint-Petersburg City Center \\ of Endocrine Surgery and Oncology, Russia
}

Correspondence: Anatoly F Romanchishen, Professor of Surgery and Oncology, Chief of Hospital Surgery and Oncology Department of Saint-Petersburg State Pediatric Medical Academy, Director of St. Petersburg Center of Endocrine Surgery and Oncology, Member of American, European, Asian, and Italian (Honorable), Associations of Endocrine Surgeons, Honored Doctor of Russian Federation, Furshtatskaya Str, 20-30, Saint-Petersburg, Russia, Phone:+79219621527, e-mail: afromanchishen@mail.ru

\begin{abstract}
Objectives: Influence of lymphatic metastases presence at immediate and follow-up results of pts surgical treatment is controversial especially for sporadic medullary thyroid carcinoma (SMTC).

Methods: 3330 thyroid cancer pts were operated on (1973-2009), among them-226 (6.8\%) medullary thyroid cancer (MTC) patients. In 11 observations MTC was appeared as a part of MEN syndrome. Sporadic character of disease was confirmed in 215 (95.1\%) cases. There were $140(80.8 \%)$ females and $33(19.2 \%)$ males (4.2:1.0) with average age $48.6 \pm 2.7$. Long-term results (1-40 years) were investigated in $173(94.2 \%)$ cases. All SMTC patients were divided into 3 groups. Group 1 made of 80 (46.2\%) T1- 3NOM0, Group 2 made of 47 (27.3\%) T1-3N1a-bM0, 3 Group made of 46 (26.6\%) T4N1a-bM0 patients.

Results:

Group 1: Organ-sparing operations were carried out in 59 (73.7\%), thyroidectomies—in $21(26.3 \%)$ patients. Multicentric tumor growth (MTG) was observed in 19.1\%: in T1 cases-5.7\%, T2-20.5\%, T3-46.7\%. All patients were undergone ipsilateral prophylactic central neck dissection (CND). Average period of postoperative observation (APPO) made $12.0 \pm 1.7 .5$ years survival rate made $98.4 \%$, $10-91.7 \%$. Local relapse of MTC was found in 1 patients (in contralateral thyroid lobe).

Group 2: Organ-sparing operations were carried out in 20 (42.6\%), thyroidectomies-27 (57.4\%) with curative central or central and lateral lymphadenectomy (LAE). MTG took place in $47.2 \% .6$ group metastases only (T1a) were found in $38.9 \%$ and CND was performed. In other cases (61.1\%) CND, lateral and mediastinal (8.3\%) LAE were performed. APPO correspondent to $10.8 \pm 2.1$ years. Repeated LAE because lymphatic metastases were carry out in 15 (31.9\%) patients through $7.2 \pm 2.4$ years in average after initial operations. Tumor relapses were not found in thyroid remnant. 5 years survival rate made $89.2 \%, 10-67.8 \%$.

Group 3: MTG was observed in 64.3\%. Central compartment group lymphatic metastases were revealed in $93.3 \%$, in 2 to 5 groups $86.7 \%$, in mediastinum-38.5\%, distant metastases—in 22.0\%. Combined operations were performed in $58.9 \%$, palliative-in 45 (26.6\%) cases. Operations were completed by Tracheostomy in 38.4\%. Adjuvant therapy (X-ray, chemotherapy) was applied in 28.9\%. Postoperative lethality made $6.5 \%$. APPO corresponded to $3.3 \pm 0.8$ (1-10 years). During first year, 8 patients have dead. 5 years survival rate made $32.4 \%$.

Conclusion: Detection of regional metastases of SMTC during the first patients presentation significantly worsen survival rate. Early diagnose of SMTC by calcitonin level detection is the best way to improve results of treatment. Organ-sparing thyroidectomies with mandatory ipsilateral prophylactics CND are justified only at T1NOMO sporadic MTC.
\end{abstract}

Keywords: Medullary thyroid carcinoma, Surgery treatment of medullary thyroid cancer.

\section{IMPORTANCE OF THE PROBLEM}

The problem of an early diagnosis of medullary thyroid cancer (MTC) remains unsolved because available diagnostic methods are very expensive. One of the most common symptoms of the C-cell cancer is diarrhea, though it can be found only in $16.0-30.0 \%$ of cases. A specific marker of primary diagnosis of MTC is serum calcitonin levels, which strongly and positively correlates with tumor spread. The most informative, but less available method of MTC diagnosis is based on the analysis of nucleic acids 
and aimed to detect the specific RET gene mutations in white blood cells from the patients and their relatives. The MTC is mostly sporadic. However, in 15.0 to $20.0 \%$ of cases, the disease is genetically determined as a dominant trait. ${ }^{1}$ Among all MTC patients, those with Multiple Endocrine Neoplasia (MEN) 2-A (Sipple's syndrome) have a better prognosis, while those with MEN 2-B syndrome have the worst prognosis. Sporadic forms of MTC have an intermediate position., ${ }^{2,3}$

Surgery is the major treatment method for MTC. However, the problem of a volume of surgery is currently unsolved, and there is no complete consensus about the strategy on both the thyroid gland and lymph nodes. Many authors consider total thyroidectomy as the only effective volume of surgery in all forms of MTC regardless of the tumor size. ${ }^{4,5}$ At the same time, there are reports that solicit organ-sparing surgery. This has been recommended because parafollicular cells are localized mostly in the apical parts of each lobe. ${ }^{6}$ There are also some recent reports about the function-sparing surgeries in cases of the C-cells microcarcinomas of the thyroid. Since there are no calcitonin-producing cells in the isthmus, excision of both lobes with preservation of isthmus has been recommended.

The most common type of dissemination in the sporadic form of MTC is lymphogenic (44.0-67.7\%), while regional metastases are found relatively rarely (37.0-50.0\%) in inheritable cases of the disease. ${ }^{7}$ The necessity to perform central neck lymphadenectomy (CNL) — removal of the 6th group of the cervical lymphnodes, is still not clear. The lymphnodes adjacent to thyroid gland contain medullary cancer metastases in $85.0 \%$ of cases, the futty tissue of the upper mediastinum - in up to $36.0 \%{ }^{8}$ There has also been an ongoing discussion about the obligatory bilateral prophylactic CNL, if lymphadenopathy was found prior to the surgery. Thus, current disagreement concerning diagnosis and treatment of patients with MTC inspired us to undertake this study.

The aim of the study was to determine the optimal volume of surgical intervention in cases of sporadic MTC based on comparison of the short- and long-term results of treatment.

\section{MATERIALS AND METHODS}

During the period from 1972 to 2009 years, 3330 patients with thyroid cancer underwent surgery at the State Pediatric Medical Academy (The Saint-Petersburg Center of
Endocrine Surgery and Oncology). The sporadic MTC was detected in 226 patients (5.8\%).

The age of the patients at the moment of diagnosis ranged from 11 to 86 years with an average of $48.5 \pm 2.7$. More than a half of the patients $(94 / 68.1 \%)$ were in the age between 40 and 70 years. The absolute majority of the patients were females (140/80.8\%) while the rest (33/19.2\%) were males resulting in male to female ratio of $1: 4.2$. It is believed that the course of the disease depends on the gender-the disease usually develops more aggressively in males. In our study, this correlation was not found. The patients were divided in three groups depending on regional and lymphogenic MTC dissemination.

The first group included 80 (40.2\%) patients with sporadic MTC without regional metastases and with minor tumor growth in the adjacent cervical tissues (T1-T3, N0, $\mathrm{M} 0$ ), who underwent surgical interventions of different volume on the thyroid. The age of these patients ranged from 23 to 83 years with an average of $52.0+3.2$. The majority of patients were females (68/85.0\%). Corresponding male to female ratio was 1:5.7.

The second group included 47 (27.2\%) sporadic MTC patients with metastases into the regional lymphnodes, but with minor tumor outgrowth into the adjacent cervical tissues (T1-T3, N1a-N1b, M0-M1), who underwent surgery of different volumes on the thyroid and the lymphatic nodes. The ages of these patients ranged from 18 to 73 years with an average of $49.6+4.8$. The majority of patients were females (38/80.8\%) also resulting in male to female ratio of 1:4.2.

The third group included 46 (26.6\%) sporadic MTC patients with significant extrathyroid tumor expansion (T4, N1a-N1b, M0-M1). In all cases, the tumor affected both lobes of the thyroid, and in $10(21.7 \%)$ cases, this was associated with lymphogenic and hematogenic dissemination. The age of these patients ranged from 11 to 86 years with an average of $61.1+2.2$. It's significantly higher $(\mathrm{P}<0.05)$ as compared to previous two groups of patients. The majority of the patients were females: 36 (76. $3 \%$ ) with corresponding male to female ratio of 1:3.6. In 34 (74.0\%) cases, the palliative surgeries were performed.

In most of the patients (115/66.9\%), the medullary cancer was the only thyroid pathology. However, in the rest $57(33.1 \%)$ cases, it was associated with other thyroid diseases, such as euthyroid goiter (45/26.2\%), diffuse toxic goiter (2/1.2\%), autoimmune thyroiditis (9/5.2\%), and acute purulent goiter (1/0.6\%). 
All patients were subjected to physical examination prior to the admittance to the clinic. We paid a particular attention to the character of complains and the family history. The conditions that could result from hyperparathyroidism and pheochromocytoma (pathological bone fractures, nephrolithiasis, and drug resistant arterial hypertension) were often found among the relatives.

The levels of parathyroid hormone, blood calcium concentration, ketosteroid urinary excretion and, if needed, genetic tests were performed in patients and their relatives to identify asymptomatic carriers of RET mutation. Genetic tests were performed in patients with confirmed disease, and also to exclude hereditary forms in patients with sporadic MTC. DNA from patient's blood and from the tumor cells obtained during surgery were used to identify mutations in RET protooncogene by the RFLP. This proto-oncogene turned into a dominant cell-transforming oncogene in all hereditary cases of C-cell carcinoma. The functional assessement of the thyroid was based on the concentration of the thyroid and pituitary hormones (tri-iodothyronine, thyroxine, calcitonin, and TSH) in blood using RIA. The calcitonin levels before surgery were elevated in all cases and ranged from 112.4 to 498.6 with the average of $296.3 \pm$ $52.4 \mathrm{mkmol} / \mathrm{l}$. In 197 cases, the ultrasound scan of the thyroid gland, abdominal area and the adrenals of patients and their relatives were performed to exclude polyneoplasia. Detailed analysis of the family history, meticulous physical examination, and on data of laboratory tests of the patients and their relatives, patients with endocrine polyneoplasia were excluded from the study.

During the last 25 years, fine-needle aspiration biopsy under ultrasound guidance has been widely used, which increased preoperational diagnosis of the MTC by up to $82.0 \%$. The biopsy was taken from several points of the thyroid, and also from regional metastases, if any of cervical lymph nodes were found enlarged. In all cases of the MTC, the diagnosis was confirmed by histological examination of the samples after the surgery.

The long-term results of the treatment (from 3-40 years) were evaluated in 173 (94.2\%) patients. They were assessed based on complains, history, TSH, thyroid hormones and calcitonin levels in blood. The volume and structure of the thyroid residual mass and the liver were examined by ultrasound scan at different times after the surgery to exclude recurrence of the tumor and/or distant metastases.
The statistical difference was evaluated by Students t-test (with $\mathrm{P}<0.05$ considered significant. Fisher exact test was used for the comparison of proportions. The survival statistics was assessed using Kaplan-Meier test.

\section{RESULTS AND DISCUSSION}

In first group of patients, the most remarkable were the complains commonly seen in nodular goiter, i.e. feeling of a lump in the throat, a node detected on the ultrasound scan, and in some instances, hoarseness and diarrhea were found.

Dysphagia, phonation, dyspnea and lymphadenopathy were found more often in patients with larger tumors and in patients with a "neglected" MTC. However, the clinical manifestations in all groups of patients could not be specific for MTC, which was diagnosed using special methods, such as fine-needle biopsy and serum calcitonin levels. During the last 15 years, fine-needle biopsy and calcitonin measurements significantly increased the probability of preoperational diagnosis of the C-cell carcinoma from $23.8 \%$ to $82.0 \%$. Monofocus carcinomas were found in 105 (58.9\%) patients. Multicentric growth of MTC was found in $73(41.1 \%)$ patients. For the surgeries on the thyroid and regional metastases, we used the method which is standard in our hospital and employs a visual control of identified recurrent and nonrecurrent laryngeal nerves, parathyroid glands, and spinal accessory nerve. The latter is necessary in the cases of lateral neck lymphadenectomy (LNL). The minimal volume of surgery used in our clinic is the ipsilateral hemithyroidectomy with CNL and partial biopsy of 3rd and 4th neck lymphatic group. The recurrent laryngeal nerve's function was assessed by an ENT physician before, and after the surgery. During the last 10 years, we use the monitoring of the recurrent and accessory nerve to make sure their function is not damaged at the end of the surgery, which is particularly important in the cases of reoperation on the thyroid.

CNL and biopsy of the cervical lymphnodes of 3rd and 4th group were performed in all MTC patients. We have found that the regional metastases, primarily affected the 6th group in $95.7 \%$, and in $63.9 \%$ - the jugular group of the neck lymph nodes. Thus, in contrary to reports by others, who doubt about the necessity of the prophylactic CNL, our data suggests that the patients may benefit from this strategy. That's why, we performed CNL in any surgical intervention on the thyroid for the sporadic intrathyroid MTC. Routine CNL under visual control of the nerves and parathyroid glands did not increase, but actually decrease to the tenfold the rate of unilateral (from 3.1 to $0.3 \%$ ) and 
bilateral (from 1.0 to $0.1 \%$ ) nerves injures, and did not increase the postoperative hypoparathyroidism $(1.0 \%$ and 0.6\%) during the period from 1998 to 2009.

LNL with the removal of paravasal adipose tissue and of groups 2 to 5 neck lymph nodes one or both sides was added, if the regional metastases were found before, during or after the surgery. At some periods of time, in our clinic, we used the surgeries of relatively the same volume, such as "Modified radical neck dissection" and "Central and lateral neck lymphadenectomy".

The character of the carcinoma growth in the thyroid tissue was assessed in all sporadic MTC cases. In the 1st group with tumors not exceeding $2 \mathrm{~cm}$ in size (T1-34 cases) monofocus tumor growth was found in $80.0 \%$, and multicentric medullar carcinomas were revealed in 4 (5.7\%). At the same time, in tumors larger than $2 \mathrm{~cm}$ (T2-34 patients), multicentric growth was found in 7 (20.5\%). In T3 carcinoma (12 cases), multicentric growth was found in $5(46.7 \%)$ cases (Table 1$)$. Thus, in patients of the 1st group, the monofocus growth was found more often ( $p<0.05$ ), if tumors were less than $2 \mathrm{~cm}$ (T1), and was seldom met, if carcinomas had larger size. The volume of surgery on the thyroid gland, in the 1st group, ranged from hemithyroidectomy (39/48.7\%), to thyroidectomy (21/ 26.3\%). Another 20(25\%) patients underwent subtotal thyroid resection.

In patients of the 2nd group, tumors size and spread corresponded to T1-in 4 (8.5\%), T2-in 16 (34.0\%), and T3-in 27 (57.5\%) patients with plural focuses of tumor grows in 23 (48.9\%) case. The most often multicentricity (59.6\%) and MTC spreading to lymphatic nodes (88.9\%) were observed in T3 cases. Macrometastases were found before and during primary operations in $48.9 \%$ and micrometastases-in others 2nd group patients during postoperative morphological examination of removal surgical specimens.

The third group, the multicentric tumor growth was found much more often, which could be explained by the characteristic intraorgan growth (within the lobe) of a large tumor, and the tendency of MTC (in tumors larger than $2 \mathrm{~cm}$ ) to lymphogenic dissemination.
Hemithyroidectomy was performed in 8 (17.0\%) patients and in 12 (25.5\%) cases, the hemithyroidectomy was followed by a subtotal resection of the second thyroid lobe.

Surgery was still the major method of treatment for "neglected" cases of carcinoma (T4), as it was in the first two groups. However, in 34 (74.0\%) cases, the surgery was palliative. In 7 (15.2\%) patients the surgery was supplemented by telegamma therapy at the dose of 4000 to 4500 Rad. Chemotherapy with Doxirubicine was used in 2 cases. The total tumor removal was performed only in 12 (26.0\%) patients.

In addition to the thyroid tissue, cervical and mediastinal adipose tissue, laryngeal and tracheal cartilage, muscular layer of the esophagus, recurrent nerve, cervical vessels and muscles involved in the tumor process were removed. Therefore, combined surgeries were performed in 27 (58.7\%) patients. Nine (19. 5\%) patients underwent emergency surgeries due to breath problems and asphyxia threat. All these latter surgeries were followed by lower tracheostomy.

A long-term follow-up was possible in 80 patients of the 1st group during the period of time from 5 to 29 years after the surgery with an average of $12.0 \pm 1.7$ years . In 47 patients of the 2nd group, long-term follow-up ranged from 5 to 26 years after the surgery with an average of $10.8 \pm 2.1$ years. Five-year survival rate, in patients of the 1st group was $98.4 \%$ (79 patients). Ten-year survival rate was $91.7 \%$ (73 patients), much higher than those reported previously $(9,10)$. Noteworthy, however, those other authors reported collective survival rate, while we report survival data for patients without regional or lymphogenic metastases. The follow-up was terminated in 4 to 17 years (mean: $8.7 \pm 2.68$ ) after the surgery. Five other (7.9\%) patients died during the time from 3 to 28 years after the surgery (on average, 15.2 \pm 3.52 ). The age of deceased patients ranged from 74 to 89 years and the causes of death were not directly linked to MTC.

Five-year survival rate in patients of the 2nd group made 89.2\% (42 patients), ten-year survival rate-in 67.0\% (32 patients), which was much less $(\mathrm{p}<0.05)$ than the survival rate in patients of the first group, and depended on the

Table 1: Character of thyroid carcinoma growth in patients of the first group (80 observations)

\begin{tabular}{|lcrrr}
\hline The tumor characteristic & $T 1(n=34)$ & $T 2(n=34)$ & $T 3(n=12) P$ & $P$ \\
\hline Monofocus growth & $30(94.3 \%)$ & $27(79.5 \%)$ & $7(53.3 \%)$ & $p<0.05$ \\
Multicentric growth & $4(5.7 \%)$ & $7(20.5 \%)$ & $5(46.7 \%)$ & $p<0.05$
\end{tabular}


Table 2: Five and ten years survival rate in patients with MTC from the first and second groups

\begin{tabular}{|lccc|} 
& Mean follow-up & Five-year survival rate & Ten-year survival rate \\
\hline The first group & $12.0 \pm 1.7 \mathrm{r}$ & $98.4 \%$ & $91.7 \%$ \\
The second group & $10.8 \pm 2.1 \mathrm{r}$ & $89.2 \%$ & $67.8 \%$
\end{tabular}

Note: the statistical significance $(P<0.05)$

severity of the regional metastatic disease at the moment of the primary tumor diagnosis. The follow-up was terminated 513 years after the surgery in $5(13.9 \%)$ patients. Other 13 (36.1\%) patients died within 14 years after the surgery (mean: $6.9+1.86)$. Three $(8.3 \%)$ of them died from causes irrelevant to MTC at the age between 70 and 81 . The rest 10 (27.8\%) patients manifested with tumor progression due to advanced regional metastases into cervical and mediastinal lymph nodes-in 9(25.0\%) patients, and also the hematogenic dissemination into the brain in one patient (2.8\%) (Table 2).

Fifteen (31.9\%) patients underwent additional surgeries on the lymph nodes at different times after the first surgery on the thyroid and lymph nodes. The recurrent regional metastases appeared within 2 to 22 years after the primary intervention, an average of $7.2 \pm 2.48$. This could be explained by late diagnosis of the carcinomas in patients of the 2nd group with extrathyroid tumor growth (T3) in 53.0\% of cases, as compared to $16.3 \%$ of patients in the second group. Out of 7 (14.9\%) patients of the 2nd group, a continuous tumor growth in the regional lymph nodes of the 2 to 5 group was found on the ipsilateral side, in 5 (10.6\%) patients - on the contralateral side, and in 3 (6.4\%) cases the metastases were found in mediastinal lymph nodes (7th group), which required additional surgery, including transsternal access.

Noteworthy, in 4 (8.5\%) patients, the regional metastases were found after organ-sparing surgeries. These were ipsilateral metastases in 3 (6.4\%) cases and contralateral in $1(2.1 \%)$ case. In these patients, a second surgery was performed to remove the residual thyroid tissue. In 9 (19.1\%) patients, regional metastases of the carcinoma were found after the thyroidectomy. Moreover, in 5 (10.6\%) cases, the metastases were contralateral or mediastinal. In all cases, the size of the carcinoma at the moment of primary surgery was larger than $4 \mathrm{~cm}$ and was associated with regional metastases into the cervical lymph nodes (T3, N1a-b, M0).

The short- and long-term results in patients with MTC with the extrathyroid tumor expansion were much worse. In $4(8.7 \%)$ cases, patients died in the clinic soon after the surgery. Seven other (15.2\%) patients died within 8 months after the surgery due to progression of the tumor growth. The rate of regional and hematogenic metastatic spread increased, when the tumor expanded into adjacent organs, which significantly affected the prognosis for these patients. The volume of surgery was also larger due to the tumor spread, and these patients underwent combined, expanded and palliative surgeries, which definitely impaired their quality of life.

The follow-up results were possible to search in 35 (76.1\%) patients of the third group within 1 to 9 years after the surgery (mean $3.3+0.84$ ). Five-year survival rate in patients of this group made $32.4 \%$ (15 patients) and appeared to be much less than that in patients of the 1st and the 2nd group ( $<0.05)$.

\section{CONCLUSION}

During the last 20 years, fine-needle aspiration biopsy and the determination of serum calcitonin levels significantly improved the chances for early diagnosis of sporadic MTC. The factors influencing to the rate of MTC getting metastatic lymph nodes metastases. The short- and long-term results analysis of the treatment allows to suggest that hemythyroidectomy could be used rarely-only in patients with intrathyroid medullary carcinoma (T1), and has to be supplemented by central cervical lymphadenectomy on the ipsilateral side up to the level of the brachiocephalic vein and the subclavicular artery. The surgery has to be accompanied with a histological evaluation of the removed tissue. If microscopic metastases and an increased level of calcitonin are found, the lateral cervical lymph dissection should be performed and the residual thyroidal mass has to be removed. If the micrometastases in the sixth group of lymph nodes are not found, the patient should be followed up using ultrasonography of the cervical lymph nodes and residual thyroid mass every 6 months. The short- and longterm results of our treatment strategy suggest that it is effective and adequate for the treatment of patients with MTC.

\section{REFERENCES}

1. Shah JP. Cancer of the head and neck. Hamilton. London. BS Decker Inc. 2001;484. 
2. Norton JA, Froome LC, Farrell RE, Wells SA (Jr). Multiple endocrine neoplasia type IIb: The most aggressive form of medullary thyroid carcinoma. Surg Clin North Am 1979;59(1): 109-18.

3. Carney JA. Familial multiple endocrine neoplasia syndromes: Components, classification, and nomenclature. J Intern Med 1998;243(6):425-32.

4. Ross DS. Long-term management of differentiated thyroid cancer. Endocr Metab Clin North Am 1990;19(3):719-39.

5. Rossi RL, Cady B, Meissner WA, et al. Nonfamilial medullary thyroid carcinoma. Am J Surg 1980;139(4):554-60.

6. Roediger WE. Thyroidectomy for nonfamilial medullary carcinoma. Brit J Surg 1976;63(5):632-42.

7. Ilyin AA, Rumyancev PO, et al. Clinic of sporadic and familial variants of medullary thyroid carcinoma. Actual problems of modern endocrinology: Thesis of all Russian Endocrinologists Congress. Saint-Petersburg 2001;305.

8. Gimm O, Ukkat J, Dralle H. Determinative factors of biochemical cure after primary and reoperative surgery for sporadic medullary thyroid carcinoma. World J Surg 1998;22(6): 562-68.

9. Brzhezovsky VZ, Shental VV, et al. Experience of medullary thyroid cancer treatment. Actual problems of modern endocrinology: Thesis of all Russian Endocrinologists Congress. Saint-Petersburg 2001;273.

10. Gharib H, McConahey WM, Tiegs RD, et al. Medullary thyroid carcinoma: Clinico-prognostic features and long-term followup of 65 patients treated during 1946 through 1970. Mayo Clin Proc 1992;67(10):934-40. 